\section{Digestive Surgery}

Amer, A. 62

Bechstein, W.O. 5, 79

Bemelmans, M.H.A. 70

Boonstra, E.A. 54

Busch, O.R. 35, 48, 70

Chandrakumaran, K. 18

Conci, S. 6

Cresswell, A.B. 18

de Boer, M.T. 54

De Jong, K.M. 70

Dejong, C.H.C. 70

Ebata, T. 23

Figueras, J. 30

Gouma, D.J. 35, 48

Guglielmi, A. 6

Habib, M. 18

Habib, N. 43

Hoekstra, L.T. 35, 48

Hui, K.Y. 62
Iacono, C. 6

Igami, T. 23

Jiao, L. 43

John, T.G. 18

Kazemier, G. 70

Liese, J. 79

Limantoro, I. 70

Lisman, T. 54

Lodewick, T.M. 70

Lopez-Ben, S. 30

Manas, D.M. 62

Moench, C. 79

Nagino, M. 23

Olde Damink, S.W.M. 70

Pai, M. 43

Porte, R.J. 54

Rees, M. 18

Reiniers, M.J. 35

Rijken, A.M. 70
Robinson, S.M. 62

Ruzzenente, A. 6

Scalzone, R. 30

Schreckenbach, T. 79

Spalding, D. 43

Stoot, J.H.M.B 70

Sugawara, G. 23

Takahashi, Y. 23

Valdegamberi, A. 6

van Dam, R.M. 70

van Gulik, T.M. 5, 35, 48

Van Hillegersberg, R. 70

van Trigt, J.D. 35

Visschers, R. 70

Welsh, F.K.S. 18

White, S.A. 62

Wong-Lun-Hing, E.M. 70

Yokoyama, Y. 23

\title{
Subject Index Vol. 29, No. 1, 2012
}

(Extended) liver resection 35

Anterior intra-hepatic approach 18

ASA classification 70

Bile leakage 48, 54

Bipolar radiofrequency 43

Cholangiocarcinoma 23

Cirrhosis 62, 79

Clamp-crush 30

Colorectal metastasis 62

Complications 70

CUSAh 30

Distal pancreatectomy 43

Endoscopic stenting 48

Extra-glissonian stapling 18

Fibrin sealant 54

Future liver remnant 79
Gallbladder cancer 23

Habib 4X 43

Hemostasis 54

Hepatectomy 23

Hospital length of stay 70

Laparoscopic liver resection 62

- - surgery 70

- resection 43

Liver 62

- failure 6

- metastasis 62

- resection $6,43,48,54$

- transection techniques 30

- tumor 70

- volume 6

Parenchymal division 30
Partial nephrectomy 43

Pedicle clamping 30

Percutaneous transhepatic biliary drainage 48

Portal vein embolization 23

Posthepatectomy liver failure 79

Prevention 79

Pringle maneuver, continuous or intermittent 35

Right hepatectomy 18

Risk analysis 79

Systematic review 70

Vascular occlusion techniques 35

\section{KARGER}

(C) 2012 S. Karger AG, Basel

Fax +41613061234 